\title{
The Effect of Methylphenidate on Neurological Soft Signs in ADHD
}

\author{
Igor Hrtanek $k^{1,2}$, Igor Ondrejka ${ }^{2}$, Ingrid Tonhajzerova ${ }^{3}$, Eva Snircova ${ }^{1,2}$, \\ Tomas Kulhan ${ }^{1,2}$, Ivan Farsky ${ }^{2,4}$, and Gabriela Nosalova ${ }^{1}$ \\ ${ }^{1}$ Department of Pharmacology, Jessenius Faculty of Medicine in Martin, Comenius University in Bratislava, Martin, Slovakia \\ ${ }^{2}$ Clinic of Psychiatry, Jessenius Faculty of Medicine and University Hospital in Martin, Comenius University in Bratislava, Martin, Slovakia \\ ${ }^{3}$ Department of Physiology, Jessenius Faculty of Medicine in Martin, Comenius University in Bratislava, Martin, Slovakia \\ ${ }^{4}$ Department of Nursing, Jessenius Faculty of Medicine in Martin, Comenius University in Bratislava, Martin, Slovakia
}

\begin{abstract}
Objective Neurological soft signs are very common in children with the attention deficit hyperactivity disorder (ADHD), and the first line medication of this disorder is methylphenidate. The aim of the study was to assess the effect of methylphenidate on the neurological soft signs in children and adolescents suffering from ADHD depending on the dose of methylphenidate.

Methods Thirty five patients with ADHD were investigated by the ADHD RS-IV parent version questionnaire and the Revised Neurological Examination for Subtle Signs before treatment adjustment and after four weeks of methylphenidate medication. The changes in hyperactivity symptomatology, neurological soft signs during therapy and the influence of the methylphenidate dose were statistically analyzed.

Results A significant decrease in hyperactivity symptomatology was found after one month of methylphenidate medication ( $\mathrm{p}=$ 0.0001 ) and significant decrease in neurological soft signs was demonstrated in 21 from a total of 26 items ( $<<0.05$ ). Correlation analysis showed no relationship between the dose of methylphenidate and the improvement of neurological soft signs. Similarly, the improvement of ADHD symptomatology had not correlation with the improvement of neurological soft signs.

Conclusion The study demonstrated the positive effect of methylphenidate on neurological soft signs in which improvement occurred independently of the dose, indicating that their progress may be due to methylphenidate treatment of any dose. The unrelated effect of methylphenidate on the attention deficit hyperactivity disorder and neurological soft signs suggest that methylphenidate might be useful in the therapy of clumsy child syndrome and in ADHD treatment of non-responders.

Psychiatry Investig 2015;12(4):545-550
\end{abstract}

Key Words Methylphenidate, ADHD, Neurological soft signs, Dosage of methylphenidate.

\section{INTRODUCTION}

Neurological soft signs (NSS) are very common in children with the attention deficit hyperactivity disorder (ADHD) in all ages, and their presence decreases with age. ${ }^{1}$ The classical definition describes NSS as a non-normative performance on a neurological examination of motor and sensory functioning in the absence of a focal lesion. ${ }^{2}$ The typical manifestations of NSS are poor coordination, speed or accuracy of limb or axial movements, including those required to keep balance, dysrhythmias and overflow. ${ }^{3}$ Despite several decades of research,

Received: December 11, 2014 Revised: February 11, 2015 Accepted: February 16, 2015 Available online: September 30, 2015

$\triangle$ Correspondence: Igor Ondrejka, MD, $\mathrm{PhD}$

Clinic of Psychiatry, Jessenius Faculty of Medicine and University Hospital in Martin, Comenius University in Bratislava, Kollarova 2, 03659 Martin, Slovakia Tel: +421-43-4203-398, Fax: +421-43-41-33-271

E-mail: ondrejka@jfmed.uniba.sk

(a) This is an Open Access article distributed under the terms of the Creative Commons Attribution Non-Commercial License (http://creativecommons.org/licenses/by$\mathrm{nc} / 3.0$ ) which permits unrestricted non-commercial use, distribution, and reproduction in any medium, provided the original work is properly cited. the origin of NSS is not clearly known and it is interpreted by abnormalities in the motor system, reflecting immaturity of neural networks. ${ }^{4}$ Although specific anatomical localization is not clearly defined, "network inhibition hypothesis" points out the central role in their interconnections among basal ganglia, cerebellum, cerebral cortex and dopamine neurotransmission which inhibit voluntary movements. ${ }^{5}$ Importantly, NSS is commonly observed in younger, developing children, while its persistence into later childhood and adolescence is pathological and have been associated with a number of neuropsychiatric and behavioral disorders such as ADHD, learning disabilities, the obsessive compulsive disorder (OCD), depression, and the conduct disorder. ${ }^{1,6}$ The presence of NSS is traditionally considered to be stable during its lifespan; however, the Lerer and Lerer study ${ }^{7}$ referred to NSS improvement during treatment with psychostimulants.

The objectification of NSS is possible through several standardized examinations such as the Examination of a Child with Minor Dysfunction, ${ }^{8}$ Movement Assessment Battery for Chil- 
dren, ${ }^{9}$ the Physical and Neurological Examination of Soft SignsPANESS, ${ }^{10}$ which was later revised, ${ }^{11}$ or one of the newer onesthe Zurich Neuromotor Assessment. ${ }^{12}$ However, most of the NSS studies in ADHD is realized by 21 items The Revised Neurological Examination of Soft Signs (revised PANESS). Patankar et al. ${ }^{1}$ found the presence of NSS in $84 \%$ of children with ADHD. In a large study, Ferrin and Vance ${ }^{13}$ confirmed a higher incidence of NSS in patients with ADHD compared to healthy children and adolescents.

The first line treatment for ADHD is currently considered to be a stimulant methylphenidate (MPH) which is relatively well-tolerated with the exception of its possible adverse effect on sleep parameters. ${ }^{14,15}$ The first pioneer study found the improvement or complete resolution of NSS in 40 children after a 60-day treatment with methylphenidate. ${ }^{7}$ The positive effect of MPH on writing and fine motor skills in general was repeatedly presented. ${ }^{16-19}$ However, none of the mentioned studies examine the correlation of the methylphenidate dose on treatment outcomes, not even the interaction between the dose of $\mathrm{MPH}$ and the improvement of NSS.

Therefore, the aim of this study was to assess the effect of methylphenidate on neurological soft signs and attention deficit hyperactivity disorder symptomatology depending on the dose of methylphenidate in children suffering from the attention deficit hyperactivity disorder.

\section{METHODS}

\section{Subjects}

The study included 35 children who were hospitalized in the Department of Psychiatry at the University Hospital in Martin between September 2012 to December 2013 with ADHD diagnosis. Patients were diagnosed according to DSM-IV-TR after being admitted to hospital by a child psychiatrist. Subsequently, every patient was examined by two independent specialists for ADHD diagnosis confirmation. The inclusion criteria were as follows: ADHD RS-IV score of $\geq 28$ before treatment; no history of neurological treatment, cramps or unconsciousness anamnesis; and no co-medication except benzodiazepines within short-term therapy. All ADHD patients were MPH naive, without a prior history of MPH treatment.

Strict exclusion criteria were used for the participants: no comorbid mental disorders; without neurological, endocrinological, metabolic, cardiovascular or other somatic diseases; without any psychotic symptoms; and no voluntary or involuntary/ behavioral symptoms potentially influencing the neurological status of ADHD patients. Twelve patients had mild transient symptoms of oppositional defiant disorder (ODD) in anamnesis which were not confirmed by detailed examination and did not meet the criteria of ODD according to DSM-IV-TR.
The study was approved by the Ethics Committee of the Jessenius Faculty of Medicine in Martin, Comenius University in Bratislava, the Slovak Republic. All the procedures performed in our study were in accordance with the ethical standards of the institutional and national research committee and with the 1964 Helsinki declaration and its later amendments or comparable ethical standards. All children/patients/guardians were carefully informed about the study protocol and informed written consent was obtained from them to participate in the study prior to examination.

\section{Drugs}

All participants were treated by orally by extended-release methylphenidate according to the Summary of Product Characteristics, the State Institute for Drug Control, the Slovak Republic. We strictly used the guidelines of treatment recommendations which define the increase of the MPH dose according to the change in ADHD symptomatology. The drug was administered once a day in the morning and the therapy started with the lowest dose, specifically 5-10 mg for younger children (6-10 years), 10-20 mg in older children (11-17 years). The final dose was specified during the first week of hospitalization and over the remaining time it was kept unchanged. This endpoint dose was also calculated in $\mathrm{mg} / \mathrm{kg}$.

\section{Protocol}

The degree of symptomatology of disease was scored twice by the ADHD RS-IV parent version questionnaire, ${ }^{20}$ which was evaluated by a psychiatrist. The first time the questionnaire was filled in during a physical admission examination, and the second time after four weeks of therapy with methylphenidate. The improvement of hyperactivity symptomatology after treatment was specified by the difference of these two scores in every patient.

Neurological soft signs were assessed analogically in the patient twice. The first examination was performed before the treatment adjustment and the second was repeated after four weeks of MPH medication. The assessment of NSS was realized by the Revised Neurological Examination for Subtle Signs ${ }^{11}$ which consists of a battery of simple tasks e.g., walk on your heels, hop on one foot, toe tapping, hand pronate/supinate, tongue wagging, place index finger on nose with eyes closed, standing with eyes closed, mouth open and tongue protruding etc. The examination focuses on the assessment of laterality, balance, gait, motor persistence and timed repetitive and patterned movements.

Lateral preference was defined as right, left and mixed. During the examination we recorded the number of errors as the prolonged time needed to complete the task, a small number or failure to do the required movements, overflow movements 
(e.g., flection of the hand and wrist while the child walks on its heels, toes and sides of feet), awkwardly posturing arms, choreioform movements, dysrhythmia, dysmetria or tremor.

The tasks were assessed based on the age of the child, which means some errors were scored or not scored (e.g., we did not score overflows with any repetitive movement in children younger than 7 years).

Finally we obtained the patient's laterality and 12 main values, namely the gait and balance total error score, dysrhythmia (quality of movement) errors, the impersistence score, the involuntary movement score, the repetitive speed of movement score, the patterned (sequenced) speed of movement score, overflow with gaits, overflow with repetitive timed movements, overflow with patterned (sequenced) timed movements, overflow in-excess-for-age, total overflow and the asymmetrical error score. From many of these values other "sub-values" are derived due to the right and left side or age. The first 11 values and their sub-values were compared before and after treatment while the asymmetrical error score was assessed according to the patient's laterality. NSS improvement after treatment was specified by the difference of all errors in neurological examination before treatment and all errors in neurological examination after treatment.

\section{Statistical analysis}

Statistical analysis was performed using the statistical software package SYSTAT 10 for Windows (SSI, Richmond, CA, USA). The Student's paired t-test was used for the parameters with Gaussian distribution and the Wilcoxon test was used for data with non-gaussian distribution to assess the changes in ADHD symptomatology and NSS before and after treatment. The correlations between variables were performed using Spearman and Pearson correlation. Specifically, the relationship was analyzed between MPH dose and ADHD improvement, between MPH dose and NSS improvement, and between ADHD improvement and NSS improvement. Correlation analysis was also used within NSS evaluation, specifically for the study of the relationship between the asymmetrical error score and the patient's laterality, twice-before and after treatment. ADHD improvement was quantified as the difference in score in the ADHD RS-IV parent version questionnaire before and after treatment, and NSS improvement was quantified as difference in all the errors in revised PANESS before and after treatment. The pvalue $<0.05$ was considered statistically significant. The data are expressed as the mean $\pm \mathrm{SD}$.

\section{RESULTS}

Our studied sample of 35 ADHD patients consisted of 29 boys and 6 girls aged between 6 and 17 years (the mean age
$11 \pm 2.12$ years). 23 children were identified with a normal IQ, 8 patients subnormal and 4 patients with mild mental retardation. The dose of MPH used was from $0.104 \mathrm{mg} / \mathrm{kg}$ to $1.364 \mathrm{mg} /$ $\mathrm{kg}$ with an average of $0.424 \mathrm{mg} / \mathrm{kg}$.

\section{ADHD symptomatology}

The ADHD RS-IV score decreased in the majority of patients $(n=32)$ after a 30-day treatment of methylphenidate while in three patients it increased. Statistically, the manifestation of ADHD symptomatology significantly decreased from a mean value of 35.9 scores before treatment to a mean value of 24.2 scores after treatment $(\mathrm{p}=0.0001)$ (Figure 1$)$.

\section{Neurological soft signs}

All 35 patients underwent and completed both of the Revised Neurological Examination for Subtle Signs. In the ADHD group, 28 right handed children, 3 left handed children and 4 children with mixed laterality were identified. The significant reduction of neurological soft signs was demonstrated in 21 from a total of 26 items during MPH therapy which are presented in Table 1. In the other items no significant differences of NSS were found (dysrhythmia left side $\mathrm{p}=0.221$, impersistence total score, $\mathrm{p}=0.056$, patterned speed of movement score left side, $\mathrm{p}=0.120$, overflow with gaits right side, $\mathrm{p}=0.861$ and

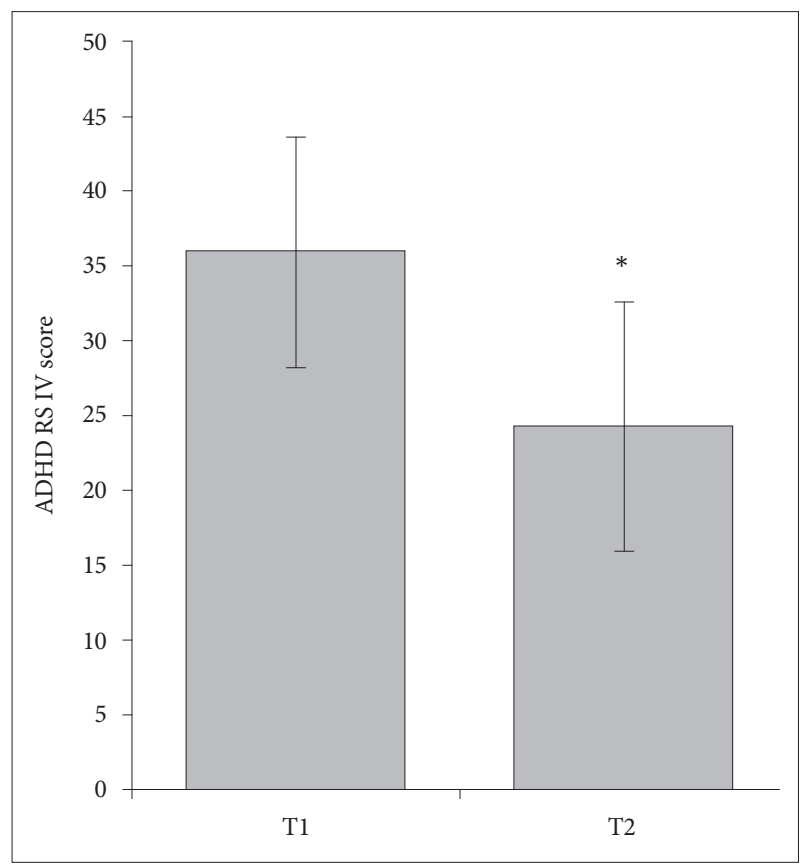

Figure 1. The influence of orally administered methylphenidate over one month in a dose $0.104-1.364 \mathrm{mg} / \mathrm{kg}$ on ADHD symptomatology. The value labeled as T1 represents the mean ADHD RSIV score before methylphenidate application and T2 represents the mean ADHD RS-IV score after methylphenidate treatment. Error bars represent standard errors of the mean $( \pm S D) .{ }^{*} p<0.05 \mathrm{com}$ pares the decrease to T1. ADHD: attention deficit hyperactivity disorder, ADHD RS-IV: ADHD Rating Scale-IV, parent version questionnaire. 
Table 1. Neurological soft signs before and after treatment

\begin{tabular}{|c|c|c|c|c|}
\hline & & Before treatment & After treatment & $\mathrm{p}$ \\
\hline Gait and balance & Total score & $8.7 \pm 4.0$ & $6.0 \pm 3.5$ & 0.0001 \\
\hline \multirow[t]{2}{*}{ Dysrhythmia } & Total score & $8.7 \pm 2.6$ & $7.2 \pm 3.1$ & 0.005 \\
\hline & Right side & $4.7 \pm 1.8$ & $3.9 \pm 1.9$ & 0.02 \\
\hline Involuntary movement score & Total score & $1.5 \pm 1.3$ & $0.7 \pm 1.0$ & 0.002 \\
\hline \multirow[t]{3}{*}{ Repetitive speed of movement } & Total score & $13.3 \pm 3.7$ & $10.9 \pm 3.9$ & 0.0001 \\
\hline & Left side & $6.1 \pm 2.5$ & $4.9 \pm 2.2$ & 0.015 \\
\hline & Right side & $7.1 \pm 2.2$ & $5.9 \pm 2.3$ & 0.003 \\
\hline \multirow[t]{2}{*}{ Patterned speed of movement score } & Total score & $1.3 \pm 1.8$ & $0.7 \pm 1.3$ & 0.009 \\
\hline & Right side & $0.6 \pm 0.9$ & $0.3 \pm 0.6$ & 0.031 \\
\hline \multirow[t]{2}{*}{ Overflow with gaits } & Asymmetrical & $2.2 \pm 1.7$ & $1.1 \pm 1.3$ & 0.001 \\
\hline & Left side & $0.2 \pm 0.5$ & $0.1 \pm 0.3$ & 0.046 \\
\hline \multirow[t]{4}{*}{ Overflow with repetitive timed movements } & Asymmetrical & $13.4 \pm 3.6$ & $10.6 \pm 3.9$ & 0.0001 \\
\hline & Left & $6.2 \pm 2.3$ & $4.9 \pm 2.2$ & 0.007 \\
\hline & Right & $7.2 \pm 2.1$ & $5.6 \pm 2.3$ & 0.0001 \\
\hline & Excessive for age & $9.0 \pm 3.6$ & $7.5 \pm 3.6$ & 0.015 \\
\hline \multirow[t]{4}{*}{ Overflow with patterned time movements } & Asymmetrical & $13.8 \pm 3.1$ & $10.2 \pm 3.9$ & 0.0001 \\
\hline & Left & $6.1 \pm 2.0$ & $4.8 \pm 2.5$ & 0.004 \\
\hline & Right & $7.7 \pm 2.0$ & $5.4 \pm 2.4$ & 0.0001 \\
\hline & Excessive for age & $9.4 \pm 2.9$ & $7.5 \pm 3.8$ & 0.002 \\
\hline Overflow in-excess-for-age & Total score & $12.7 \pm 4.6$ & $9.9 \pm 4.4$ & 0.002 \\
\hline Total overflow & Total score & $24.0 \pm 7.1$ & $18.4 \pm 7.7$ & 0.0001 \\
\hline
\end{tabular}

The data are expressed in mean $\pm \mathrm{SD}$

no overflow with gaits excessive for age was found). The correlation of the asymmetrical error score and laterality was studied and the number of errors in the NSS examination was significantly higher on the predominant side before $(\mathrm{r}=0.379, \mathrm{p}=$ $0.0248)$ and also after treatment $(\mathrm{r}=0.345, \mathrm{p}=0.0424)$.

\section{MPH dose and the efficacy of treatment}

The relationship between NSS improvement, MPH dose and the improvement of the degree of ADHD symptomatology was studied. Correlation analysis found no significant relationships in following parameters: between MPH dose and ADHD improvement $(\mathrm{r}=-0.018, \mathrm{p}=0.918)$, MPH dose and NSS improvement $(\mathrm{r}=-0.060, \mathrm{p}=0.732)$ and NNS improvement and ADHD improvement $(r=-0.129, p=0.46)$.

\section{DISCUSSION}

The major finding of this study was a significant improvement of ADHD symptomatology and NSS during four weeks of $\mathrm{MPH}$ treatment without any significant relationships to the $\mathrm{MPH}$ dose. ADHD improvement was not significantly related to the improvement of NSS.

Our results are in agreement with the other studies that re- vealed a positive effect of methylphenidate on ADHD symptomatology. ${ }^{21-24}$ With respect to NSS, the improvement or complete resolution in children with $\mathrm{ADHD}$ was firstly demonstrated by Lerer and Lerer ${ }^{7}$ after a 60-day MPH treatment. Another study demonstrated the effectiveness of persistent administration of MPH on deficits in motor timing in ADHD children and repeatedly showed new possibilities of this medication from the domain of attentional and inhibitory functions to the domain of motor functions. ${ }^{25}$ The results of our study confirmed these findings, but in contrast, the presented study included more adolescent patients and used a different type of MPH-extended-release methylphenidate.

The specific CNS region underlying NSS symptomatology is still not fully explained. All errors in particular items of NSS examination are related with planning and controlling action. The motor planning is linked to the pre-supplementary motoric area (pre-SMA) and connections between the prefrontal cortex, basal ganglia and cerebellum. ${ }^{26,27}$ Motor control depends on the cerebellum, especially with connections to preSMA and the primary motor cortex. ${ }^{28}$ From this perspective, the frequently examined age-inappropriate overflow movements are probably a reflection of immaturity of the cortical systems involved in automatic inhibition. ${ }^{29}$ Furthermore, the 
effect of MPH in the treatment of NSS is supposed on the dopamine reuptake in basal ganglia, cerebellum and cerebral cortex interconnection. ${ }^{5}$ Therefore, it could be considered that MPH acts in similar regions and may improve NSS.

To the best of our knowledge, the relationship between the dose of MPH and the efficacy of treatment of NSS were not sufficiently described. Our results did not find a relationship between the MPH dose and the improvement of NSS as well as ADHD symptoms. The findings could have been distorted by the dosage of $\mathrm{MPH}$, not random, but corresponding to the clinical state of the patients during the first week (titration of the MPH dose according to the manifestation of ADHD symptoms). However, we did not take into account the severity of NSS symptoms during the dosing of MPH and a higher dose did not cause a greater reduction of NSS. For this reason we could hypothesize that the dose of MPH is not principal in the efficacy of NSS treatment.

However, individual sensitivity to MPH could play an important role. It is well-known that this personal sensitivity is known in the treatment of ADHD in which some children respond and others do not respond to MPH. ${ }^{30}$ Song et al. ${ }^{31}$ found that younger patients need a large dose to control ADHD symptoms. Clatworthy et al. ${ }^{32}$ showed that effect of MPH on reversal learning and spatial working memory depends on individual differences in dopamine receptors with availability in post-commissural caudate and in the ventral striatum. Given these findings we might speculate that different personal sensitivity to methylphenidate also affects NSS treatment.

Interestingly, we found that the effect of MPH on independent NSS and ADHD symptomatology is different in the same sample of patients. We demonstrated no relationship between the improvement of NSS and ADHD symptomatology. Even a negative correlation in our results indicates that less improvement of ADHD symptomatology is connected with higher improvement of NSS and vice-versa. We might speculate that different MPH action on ADHD and NSS could be caused by dopamine reuptake in independent areas-in the prefrontal cortex and striatal circuits in the domain of attentional and inhibitory functions and in basal ganglia, cerebellum and cerebral cortex interconnection in the domain of motor functions. ${ }^{5,33}$ Other reasons for the independent improvement of NSS and ADHD could be the difference in dynamic reactivity during a time period (e.g., a faster effect of MPH on ADHD symptomatology than on NSS or vice-versa), or different severity of these symptomatologies before treatment (e.g., more exaggerated NSS than ADHD symptoms or vice-versa).

Our findings showed that MPH could help in the treatment of clumsy child syndrome, which is very often part of a complex symptomatology of ADHD. It seems that the dose of $\mathrm{MPH}$ does not play a dominant role in this improvement and we should always predict reduction in NSS during MPH treatment, independent of dose. According to our results of a non-significant relationship between the improvement of ADHD core symptomatology and NSS we could hypothesize that MPH might have an effect on clumsy child syndrome not only in ADHD responders, but also in non-responders. The revised PANESS is easily achievable and an inexpensive examination with high reliability, ${ }^{34}$ and it can help to assess the response to treatment in a specific group of ADHD patients with exaggerated clumsy child syndrome.

We suggest that further research with a large number of patients is needed, in particular with respect to the NSS examination in ADHD non-responders to methylphenidate. Furthermore, we assume that longitudinal study into adult-age patients is needed due to their potential NSS manifestation. Finally, our findings may be confronted with further studies using neuroimaging methods, which could be beneficial for understanding this issue better.

In conclusion, a significant effect of MPH was demonstrated on ADHD symptoms and NSS with both improvements reached independent of each other. Moreover, ADHD and NSS improved independently to the dose of MPH, indicating that the dosage could play no significant role in the efficacy of treatment. We suggest that these findings may help elucidate the $\mathrm{MPH}$ effect on the complex ADHD-neurological interaction.

\section{Acknowledgments}

This work was supported by the Scientific Grant Agency of The Slovak Republic (VEGA) 1/0087/14.

\section{REFERENCES}

1. Patankar VC, Sangle JP, Shah HR, Dave M, Kamath RM. Neurological soft signs in children with attention deficit hyperactivity disorder. Indian J Psychiatry 2012;54:159-165.

2. Shafer SQ, Shaffer D, O'Connor PA, Stokman CJ. Hard Thoughts on Neurological “Soft Signs”. In: Rutter M, Editor. Developmental Neuropsychiatry. New York: Guilford Press, 1983, p.133-143.

3. Martins I, Lauterbach M, Slade P, Luis H, De Rouen T, Martin M, et al. A longitudinal study of neurological soft signs from late childhood into early adulthood. Dev Med Child Neurol 2008;50:602-607.

4. Mostofsky SH, Newschaffer CJ, Denckla MB. Overflow movements predict impaired response inhibition in children with ADHD. Percept Mot Skills 2003;97:1325-1331.

5. Pasini A, D'Agati E. Pathophysiology of NSS in ADHD. World J Biol Psychiatry 2009;10:495-502.

6. Larson JC, Mostofsky SH, Goldberg MC, Cutting LE, Denckla MB, Mahone EM. Effects of gender and age on motor exam in typically developing children. Dev Neuropsychol 2007;32:543-562.

7. Lerer RJ, Lerer MP. The effects of methylphenidate on the soft neurological signs of hyperactive children. Pediatrics 1976;57:521-525.

8. Touwen BC, Sporrel T. Soft signs and MBD. Dev Med Child Neurol 1979;21:528-530.

9. Henderson SE, Hall D. Concomitants of clumsiness in young schoolchildren. Dev Med Child Neurol 1982;24:448-460.

10. Guy W. Physical and Neurological Examination for Soft Signs (PANESS). In: Guy W, Editor. ECDEU Assessment Manual for Psychophar- 
macology. Rockville, MD: National Institute of Mental Health, 1976, p. 383-393.

11. Denckla MB. Revised neurological examination for subtle signs (1985). Psychopharmacol Bull 1985;21:773-800.

12. Largo RH, Fischer JE, Caflisch JA. Zurich Neuromotor Assessment. Zürich: AWE Verlag; 2002.

13. Ferrin M, Vance A. Examination of neurological subtle signs in ADHD as a clinical tool for the diagnosis and their relationship to spatial working memory. J Child Psychol Psychiatry 2012;53:390-400.

14. Sangal RB, Owens J, Allen AJ, Sutton V, Schuh K, Kelsey D. Effects of atomoxetine and methylphenidate on sleep in children with ADHD. Sleep 2006;29:1573-1585.

15. Lee SH, Seo WS, Sung HM, Choi TY, Kim SY, Choi SJ, et al. Effect of methylphenidate on sleep parameters in children with ADHD. Psychiatry Investig 2012;9:384-390.

16. Senger EY, Hallum G. Methylphenidate in children with minimal brain dysfunction: effects on attention span, visual motor skills and behavior. Curr Ther Res Clin Exp 1974;16:635-641.

17. Lerer RJ, Lerer MP, Artner J. The effects of methylphenidate on the handwriting of children with minimal brain dysfunction. J Pediatr 1977;91:127132 .

18. Tirosh E, Elhasid R, Kamah SC, Cohen A. Predictive value of placebo methylphenidate. Pediatr Neurol 1993;9:131-133.

19. Peeples EE, Searls DT, Wellingham-Jones P. Attention-deficit hyperactivity disorder: a longitudinal case study of handwriting characteristics. Percept Mot Skills 1995;81:1243-1252.

20. DuPaul GJ, Power TJ, Anastopoulos AD, Reid R. ADHD Rating ScaleIV: Checklists, Norms, and Clinical Interpretation. New York: Guilford Press; 1998.

21. Whalen CK, Henker B. Social impact of stimulant treatment for hyperactive children. J Learn Disabil 1991;24:231-241.

22. Schachar RJ, Tannock R, Cunningham C, Corkum PV. Behavioral, situational, and temporal effects of treatment of ADHD with methylphenidate. J Am Acad Child Adolesc Psychiatry 1997;36:754-763.

23. Musten LM, Firestone P, Pitserman S, Bennett S, Mercer J. Effects of methylphenidate on preschool children with ADHD: cognitive and behavioral functions. J Am Acad Child Adolesc Psychiatry 1997;36:1407-1415.

24. Abikoff H, Hechtnab L, Klein RG, Weiss G, Fleiss K, Etcovitch J, et al.
Symptomatic improvement in children with ADHD treated with longterm methylphenidate and multimodal psychosocial treatment. J Am Acad Child Adolesc Psychiatry 2004;43:802-811.

25. Rubia K, Noorloos J, Smith A, Gunning B, Sergeant J. Motor timing deficits in community and clinical boys with hyperactive behavior: the effect of methylphenidate on motor timing. J Abnorm Child Psychol 2003; 31:301-313.

26. Hoshi E, Tanii J. Differential roles of neuronal activity in the supplementary and presupplementary motor areas: from information retrieval to motor planning and execution. J Neurophysiol 2004;92:3482-3499.

27. Doya K. Complementary roles of basal ganglia and cerebellum in learning and motor control. Curr Opin Neurobiol 2000;10:732-739.

28. Sakai K, Hikosaka O, Takino R, Miyauchi S, Nielsen M, Tamada T. What and when: parallel and convergent processing in motor control. J Neurosci 2000;20:2691-2700.

29. Mostofsky SH, Newschaffer CJ, Denckla MB. Overflov movements predict impaired response inhibition in children with ADHD. Percept Mot Skills 2003;97:1315-1331.

30. Newcorn JH, Kratochvil CHJ, Allen AJ, Casat CHD, Ruff DD, Moore RJ, et al. Atomoxetine and osmotically released methylphenidate for the treatment of attention deficit hyperactivity disorder: acute comparison and differential Response. Am J Psychiatry 2008;165:721-730.

31. Song DH, Choi S, Joung YS, Ha EH, Kim BN, Shin YJ, et al. Titrating optimal dose of osmotic-controlled release oral delivery (OROS)-methylphenidate and its efficacy and safety in Korean Children with ADHD: A multisite open labeled study. Psychiatry Investig 2012;9:257-262.

32. Clatworthy PL, Lewis SJ, Brichard L, Hong YT, Izquierdo D, Clark L, et al. Dopamine Release in dissociable striatal subregions predicts the different effects of oral methylphenidate on reversal learning and spatial working memory. J Neurosci 2009;29:4690-4696.

33. Goldman-Rakic PS, Cools AR, Srivastava K. The prefrontal landscape: implications of functional architecture for understanding human mentation and the central executive [and discussion]. Phil Trans R Soc Lond B 1996;351:1445-1453.

34. Gusstafson P, Svedin CG, Ericsson I, Lindén CH, Karlsson M, Therlnlund G. Reliability and validity of the assessment of neurological soft-signs in children with and without attention-deficit-hyperactivity disorder. Dev Med Child Neurol 2010;52:364-370. 